\title{
Chapter 1 Bernstein-type polynomials on several intervals
}

\author{
J. Szabados ${ }^{\star}$
}

Dedicated to the memory of Professor Qazi Ibadur Rahman

\begin{abstract}
We construct the analogues of Bernstein polynomials on the set $J_{s}$ of $s$ finitely many intervals. Two cases are considered: first when there are no restrictions on $J_{s}$, and then when $J_{s}$ has a so-called T-polynomial. On such sets we define approximating operators resembling the classic Bernstein polynomials. Reproducing and interpolation properties, as well as estimates for the rate of convergence are given.
\end{abstract}

Keywords: Bernstein polynomial, T-polynomial, set of intervals, rate of convergence

2000 Mathematics Subject Classification: 41A10, 41A25

\subsection{Introduction}

For any $s \geq 1$ let

$$
0=a_{1}<b_{1}<\cdots<a_{s}<b_{s}=1
$$

be a finite partition of the interval $[0,1]$, let $I_{j}:=\left[a_{j}, b_{j}\right], j=1, \ldots, s$, and let

$$
J_{s}:=\bigcup_{j=1}^{s} I_{j}, \quad I_{j}:=\left[a_{j}, b_{j}\right]
$$

J. Szabados

Alfréd Rényi Institute of Mathematics,

P.O.B. 127, H-1364 Budapest, Hungary

e-mail: szabados.jozsef@renyi.mta.hu

* Research supported by OTKA Grant No. K111742. 
be the corresponding set of $s$ pairwise disjoint intervals, and denote by $\Pi_{n}$ the set of polynomials of degree at most $n$. Denote by $C\left(J_{s}\right)$ the set of continuous functions on $J_{s}$.

Some basic problems of approximation theory, like Markov-Bernstein inequalities or Lagrange interpolation have been considered for functions $f \in C\left(J_{s}\right)$; see for example [4] or [2]. It is equally important to establish analogues of classic operators on this set. In this context we will be concerned with generalizations of Bernstein polynomial.

\subsection{The general case}

The simplest construction for a general set $J_{s}$ is the following. If $f \in C\left(J_{s}\right)$ is not differentiable, then extend it to $[0,1] \backslash J_{s}$ by defining it as a linear function in each $\left[b_{j}, a_{j+1}\right], j=1, \ldots, s-1$, continuously connecting the different parts of the original function. If $f^{\prime} \in C\left(J_{s}\right)$ then in each $\left[b_{j}, a_{j+1}\right], j=1, \ldots, s-1$, define $f$ as the third degree Hermite polynomial satisfying

$H_{j}\left(b_{j}\right)=f\left(b_{j}\right), H_{j}^{\prime}\left(b_{j}\right)=f^{\prime}\left(b_{j}\right), H_{j}\left(a_{j+1}\right)=f\left(a_{j+1}\right), H_{j}^{\prime}\left(a_{j+1}\right)=f^{\prime}\left(a_{j+1}\right)$,

$1 \leq j \leq s-1$. Then evidently the modulus of continuity $\omega(\tilde{f}, t) \leq c \omega(f, t)$ in the first case, and $\omega\left(\tilde{f}^{\prime}, t\right) \leq c \omega\left(f^{\prime}, t\right)$ in the second case. Thus considering the ordinary Bernstein polynomials for the extended function $\tilde{f}$ on $[0,1]$, we obtain the usual error estimates $c \omega(f, 1 / \sqrt{n})$ and $\omega\left(f^{\prime}, 1 / \sqrt{n}\right) / \sqrt{n}$ in the corresponding cases, respectively. (In the latter case besides function values, derivative values at the endpoints $I_{j}$ of the function also appear in the construction.)

This method has two disadvantages. First, in the classic case the difference between the number of data used in the construction and the degree of the Bernstein polynomials is 1, while here it is $c n$. Second, the classic Bernstein polynomials interpolate at the endpoints of the interval, while here not.

\subsection{The case of T-polynomials}

It is well-known that given arbitrary positive integer $m$, a set $J_{s}$ always has a so-called Chebyshev polynomial of degree $m$, i.e. a polynomial which attains its maximum and minimum on $J_{s}$ at consecutive $m+1$ points in $J_{s}$. If, in addition, this polynomial attains its maximum or minimum on $J_{s}$ at the endpoints of $I_{j}, j=1, \ldots, s$, then we say that this is a T-polynomial. Of course, this additional property does not hold for all sets $J_{s}$. It was $\mathrm{F}$. Peherstorfer who introduced this notion, and thoroughly investigated its existence and properties (see [5], [6]). 
It will be convenient to assume that a T-polynomial has a minimum 0 and maximum 1 on $J_{s}$.

Assume that $J_{s}$ has a T-polynomial $p(x) \in \Pi_{m}, m \geq s$ normalized such that $p(0)=0$. For $n \in \mathbb{N}$, let $x_{k 1}<\cdots<x_{k m_{k}}$ be defined by

$$
p\left(x_{k i}\right)=\frac{k}{n}, \quad i=1, \ldots, m_{k} ; \quad k=0, \ldots, n
$$

where

$$
m_{k}= \begin{cases}m+s-\left[\frac{m+s}{2}\right], & \text { if } \quad k=0, \\ m, & \text { if } \quad k=1, \ldots, n-1, \\ {\left[\frac{m+s}{2}\right],} & \text { if } \quad k=n .\end{cases}
$$

The existence of such $x_{k i}$ 's follows from the properties of T-polynomials (they are monotone between two adjacent extremal values).

For an arbitrary $f(x) \in C\left(J_{s}\right)$, let

$$
L_{k}(f, x)=\sum_{i=1}^{m_{k}} f\left(x_{k i}\right) \ell_{k i}(x) \in \Pi_{m_{k}-1}, \quad k=0,1, \ldots, n,
$$

be the Lagrange interpolation polynomial with respect to the nodes $x_{k i}$. Here $\ell_{k i}(x) \in \Pi_{m_{k}-1}$ are the fundamental polynomials with the property

$$
\ell_{k i}\left(x_{k j}\right)=\delta_{i j}, \quad i, j=1, \ldots, m_{k}, \quad k=0,1, \ldots, n .
$$

Consider the discrete linear operator

$$
B_{n}(f, x):=\sum_{k=0}^{n} L_{k}(f, x) b_{n k}(p(x)), \quad x \in J_{s},
$$

where

$$
b_{n k}(x)=\left(\begin{array}{l}
n \\
k
\end{array}\right) x^{k}(1-x)^{n-k}, \quad k=0, \ldots, n,
$$

are the fundamental functions of the Bernstein polynomials. Evidently, $B_{n}(f, x) \in \Pi_{m n+m-1}$, and there are $m n+s$ function values used in the construction of the operator. This means that the difference between the number of function values and the degree of the operator is $m-s+1$, i.e. independent of $n$, just like in case of the classic Bernstein polynomials. In this respect they are better than the polynomials defined in the previous section.

Although this is not a positive operator, it still has a bounded norm; this will follow from Theorem 1 below.

We mention two properties of this operator. The first one is about reproducing polynomials: 


$$
B_{n}(q, x) \equiv q(x) \quad \text { if } \quad q \in \Pi_{m_{n}-1} \quad \text { or } \quad q=p .
$$

Namely, if $q \in \Pi_{m_{n}-1}$, then $L_{k}(q, x) \equiv q(x), k=0,1, \ldots, n$ by the reproducing property of Lagrange interpolation, and thus

$$
B_{n}(q, x)=q(x) \sum_{k=0}^{n} b_{n k}(p(x))=q(x) .
$$

And if $q=p$, then $L_{k}(p, x) \equiv \frac{k}{n}, k=0,1, \ldots, n$, whence

$$
B_{n}(p, x)=\sum_{k=0}^{n} \frac{k}{n} b_{n k}(p(x))=p(x)
$$

since the classic Bernstein polynomials reproduce linear functions. Again, this is better than the corresponding reproducing property of the classic Bernstein polynomials (which reproduce only linear functions).

The second property is about interpolation:

$$
B_{n}\left(f, x_{k i}\right)=f\left(x_{k i}\right), \quad i=1, \ldots, m_{k} ; \quad k=0 \text { or } n .
$$

Namely, $p\left(x_{0 i}\right)=0, i=1, \ldots, m_{0}$, and thus

$$
B_{n}\left(f, x_{0 i}\right)=L_{0}\left(f, x_{0 i}\right)=f\left(x_{0 i}\right), \quad i=1, \ldots, m_{0} .
$$

Similarly for the $x_{n i}$ 's. Notice that the polynomials defined in the previous section interpolate only at 0 and 1 .

Now we state a pointwise convergence estimate. Let

$$
\varphi(x)=\sqrt{\left(x-a_{j}\right)\left(b_{j}-x\right)} \quad \text { if } \quad x \in I_{j}, j=1, \ldots, s,
$$

and define the Ditzian-Totik modulus of continuity (cf. [1], Ch. 1) as

$$
\omega_{\varphi}(f, t)=\sup _{0<h \leq t}\left\|\Delta_{h \varphi(x)} f(x)\right\|_{J_{s}}
$$

where the difference is meant to be zero if any of the arguments is outside $J_{s}$, and we assume that $t$ is so small that both $x \pm \varphi(x)$ fall into the same interval $I_{j}$. Further let

$$
V(f)=\sup _{x, y \in J_{s}}|f(x)-f(y)| .
$$

Theorem 1. For an arbitrary $f \in C\left(J_{s}\right)$ we have

$$
\left\|f(x)-B_{n}(f, x)\right\|_{J_{s}} \leq c \omega_{\varphi}\left(f, \frac{1}{\sqrt{n}}\right)+c \frac{V(f)}{\sqrt{n}} .
$$


(Here and in what follows, $c$ will always denote a positive constant depending on $J_{s}$ and $m$, but independent of $n$, not necessarily the same at each occurrence.) Note that the second term in the error estimate cannot be dropped, since for functions which are constant on each interval $I_{j}$, the modulus of continuity is zero, while the operator does not reproduce such functions.

For the proof we need the following

Lemma 1. Let $(\alpha, \beta)$ be one of the pairs of numbers $(0,1),(1 / 2,1),(1,1)$, $(1,2),(3 / 2,2)$. Then

$$
\sum_{k=1}^{[n / 2]}\left(\frac{n}{k}\right)^{\alpha}\left|x-\frac{k}{n}\right|^{\beta} b_{n k}(x) \leq\left\{\begin{array}{lll}
c\left(\frac{x}{n}\right)^{\frac{\beta-\alpha}{2}}, & \text { if } & 0 \leq x \leq 1 / n \\
c \frac{x^{\beta / 2-\alpha}}{n^{\beta / 2}}, & \text { if } & 1 / n \leq x \leq 1 .
\end{array}\right.
$$

Proof. By Cauchy-Schwarz inequality we get for $0 \leq x \leq 1 / n$,

$$
\begin{aligned}
T:=\sum_{k=1}^{[n / 2]}\left(\frac{n}{k}\right)^{\alpha}\left|x-\frac{k}{n}\right|^{\beta} b_{n k}(x) & \leq\left(\sum_{k=1}^{[n / 2]}\left(\frac{n}{k}\right)^{2 \alpha}\left(x-\frac{k}{n}\right)^{2 \beta} b_{n k}(x)\right)^{1 / 2} \\
& \leq\left(\sum_{k=1}^{[n / 2]}\left(x-\frac{k}{n}\right)^{2 \beta-2 \alpha} b_{n k}(x)\right)^{1 / 2} .
\end{aligned}
$$

Now we use the inequalities

$$
\sum_{k=0}^{n}\left|x-\frac{k}{n}\right|^{2 \gamma} b_{n k}(x) \leq c\left(\frac{x}{n}\right)^{\gamma}
$$

where $\gamma=1 / 2$ or 1 and $0 \leq x \leq 1$, or $\gamma=2$ and $1 / n \leq x \leq 1$. These follow from well-known relations (cf. Lorentz [3], p. 14). Hence we obtain the first statement of the lemma.

Next let $1 / n \leq x \leq 1$. We obtain, using again (1.5),

$$
\begin{aligned}
T & \leq\left(\sum_{k=1}^{[n / 2]}\left(\frac{n}{k}\right)^{2 \alpha}\left(x-\frac{k}{n}\right)^{2 \beta} b_{n k}(x)\right)^{1 / 2} \leq \frac{1}{x^{\alpha}}\left(\sum_{k=1}^{[n / 2]}\left(x-\frac{k}{n}\right)^{2 \beta} b_{n+2 \alpha, k}(x)\right)^{1 / 2} \\
& \leq \frac{c}{x^{\alpha}}\left(\sum_{k=1}^{[n / 2]}\left(x-\frac{k}{n+2 \alpha}\right)^{2 \beta} b_{n+2 \alpha, k}(x)\right)^{1 / 2}+c \frac{1}{n^{\beta-\alpha}} \leq c \frac{x^{\beta / 2-\alpha}}{n^{\beta / 2}} \cdot
\end{aligned}
$$

Proof of Theorem 1. Let $x \in(\xi, \eta) \subset I_{j}=\left[a_{j}, b_{j}\right]$ be such that $p(\xi)=$ $0, p(\eta)=1$ and $p^{\prime}(x)>0$ (the case $p^{\prime}(x)<0$ can be handled similarly). Let 
$p\left(x_{k t}\right)=\frac{k}{n}$ where $x_{k t} \in[\xi, \eta]$. Let

$$
S_{k}= \begin{cases}\{t\}, & \text { if } \quad \xi=a_{j}, \\ \{t-1, t\}, & \text { if } \xi>a_{j} .\end{cases}
$$

Since both operators $L_{k}$ and the classic Bernstein polynomials reproduce constants, we get

$$
\begin{aligned}
\left|f(x)-B_{n}(f, x)\right| \leq & \sum_{k=0}^{n} \sum_{i=1}^{m_{k}}\left|f(x)-f\left(x_{k i}\right)\right| \cdot\left|\ell_{k i}(x)\right| b_{n k}(p(x)) \\
\leq & c \sum_{k=0}^{n}\left\{\omega_{\varphi}\left(f, \frac{1}{\sqrt{n}}\right) \sum_{i \in S_{k}}\left[\frac{\sqrt{n}}{\varphi(x)}\left(x-x_{k i}\right)+1\right]\left|\ell_{k i}(x)\right|\right. \\
& \left.+V(f) \sum_{i \notin S_{k}}\left|\ell_{k i}(x)\right|\right\} b_{n k}(p(x)) .
\end{aligned}
$$

Here we used the inequality $\varphi\left(\frac{x+x_{k i}}{2}\right) \geq c \varphi(x), i \in S_{k}$.

We estimate the right hand side sum for $0 \leq k \leq n / 2$; the other part can be handled similarly. Then it is sufficient to consider the case $0 \leq p(x) \leq 4 / 5$, since for $4 / 5 \leq p(x) \leq 1$ we get

$$
\begin{gathered}
\sum_{k=0}^{[n / 2]}\left(\frac{n}{k}\right)^{\alpha} b_{n k}(p(x)) \leq n^{\alpha}(1-p(x))^{n / 2} \sum_{k=0}^{n}\left(\begin{array}{l}
n \\
k
\end{array}\right) p(x)^{k} \\
\leq n^{\alpha} 2^{n}(1 / 5)^{n / 2}=n^{\alpha}(4 / 5)^{n / 2}
\end{gathered}
$$

This geometric convergence to zero implies that all similar sums to be handled subsequently are minor compared to the estimates for $0 \leq p(x) \leq 4 / 5$. Also, it is sufficient to consider the case when $\eta<b_{j}$, since $\eta=b_{j}$ corresponds to the case $\xi=a_{j}$.

Let first $k=0$. Then we have $\left|\ell_{0 i}(x)\right| \leq c, i=0,1, \ldots, m_{0}$, and

$$
\frac{\left(x-x_{0 i}\right)\left|\ell_{0 i}(x)\right|}{\varphi(x)} \leq c \frac{x-x_{0 t}}{\varphi(x)} \leq c \sqrt{p(x)}, \quad i=1, \ldots, m_{0} .
$$

Hence

$$
\left(\frac{\sqrt{n}}{\varphi(x)}\left(x-x_{0 i}\right)+1\right)\left|\ell_{0 i}(x)\right| b_{n 0}(p(x)) \leq c\left(\sqrt{n p(x)}(1-p(x))^{n}+c \leq c .\right.
$$

Now let $1 \leq k \leq n / 2$. In order to complete the proof of Theorem 1 we need to prove the following estimates: 


$$
\begin{gathered}
A:=\sum_{k=1}^{[n / 2]} \sum_{i \in S_{k}}\left|\ell_{k i}(x)\right| b_{n k}(p(x)) \leq c, \\
B:=\sum_{k=1}^{[n / 2]} \sum_{i \in S_{k}}\left|x-x_{k i}\right| \cdot\left|\ell_{k i}(x)\right| b_{n k}(p(x)) \leq \frac{\varphi(x)}{\sqrt{n}}, \\
C:=\sum_{k=1}^{[n / 2]} \sum_{i \notin S_{k}}\left|\ell_{k i}(x)\right| b_{n k}(p(x)) \leq \frac{c}{\sqrt{n}} .
\end{gathered}
$$

We distinguish two cases.

Case 1: $S_{k}=\{t\}$. Then $\left|\ell_{k t}(x)\right| \leq c$, whence $A \leq c \sum_{k=1}^{[n / 2]} b_{n k}(p(x)) \leq c$. On the other hand, $\left|x-x_{k t}\right| \leq c\left|p(x)-\frac{k}{n}\right|$, therefore by Lemma 1 applied with $\alpha=0, \beta=1$ yields $B \leq c \sum_{k=1}^{[n / 2]}\left|x-x_{k t}\right| b_{n k}(p(x)) \leq c \sum_{k=1}^{[n / 2]}\left|p(x)-\frac{k}{n}\right| b_{n k}(p(x)) \leq c \sqrt{\frac{p(x)}{n}} \leq c \frac{\varphi(x)}{\sqrt{n}}$.

Finally, using $\left|\ell_{k i}(x)\right| \leq c \sqrt{\frac{n}{k}}\left|x-x_{k t}\right|, i \notin S_{k}$, and Lemma 1 with $\alpha=$ $\frac{1}{2}, \beta=1$,

$$
C \leq c \sum_{k=1}^{[n / 2]} \sqrt{\frac{n}{k}}\left|x-x_{k t}\right| b_{n k}(p(x)) \leq c \sum_{k=1}^{[n / 2]} \sqrt{\frac{n}{k}}\left|p(x)-\frac{k}{n}\right| b_{n k}(p(x)) \leq \frac{c}{\sqrt{n}} .
$$

Case 2: $S_{k}=\{t-1, t\}$. Then

$$
\begin{gathered}
\left|\ell_{k t}(x)\right| \leq c \frac{x-x_{k, t-1}}{x_{k t}-x_{k, t-1}} \leq 1+c \sqrt{\frac{n}{k}}\left|x-x_{k t}\right|, \\
\left|\ell_{k, t-1}(x)\right| \leq c \frac{\left|x-x_{k t}\right|}{x_{k t}-x_{k, t-1}} \leq c \sqrt{\frac{n}{k}}\left|x-x_{k t}\right| . \\
\left|\ell_{k i}(x)\right| \leq c \frac{\left|x-x_{k t}\right|\left(x-x_{k, t-1}\right)}{x_{k i}-x_{k, i \pm 1}} \leq c\left|x-x_{k t}\right|+\sqrt{\frac{n}{k}}\left(x-x_{k t}\right)^{2}, \quad i \notin S_{k} .
\end{gathered}
$$

We also need the inequalities 


$$
\left|x-x_{k t}\right| \leq \begin{cases}c \sqrt{\frac{n}{k}}\left(p(x)-\frac{k}{n}\right), & \text { if } \quad 1 \leq k \leq n \min (p(x), 1 / 2), \\ c \min \left(\sqrt{\frac{k}{n}}, \frac{1}{\sqrt{p(x)}}\left(\frac{k}{n}-p(x)\right)\right) & \text { if } \quad n \min (p(x), 1 / 2)<k \leq n / 2,\end{cases}
$$

and

$$
0<x-x_{k, t-1} \leq c\left|x-x_{k t}\right|+c \sqrt{\frac{k}{n}} .
$$

These relations can be easily seen by using the mean value theorem and considering that $p^{\prime}(x) \sim \sqrt{p(x)}$.

Using Lemma 1 with $\alpha=\beta=1$ we have

$$
\begin{gathered}
A \leq c \sum_{k=1}^{[n / 2]}\left(1+\sqrt{\frac{n}{k}}\left|x-x_{k t}\right|\right) b_{n k}(p(x)) \\
\leq c+c \sum_{k=1}^{[n \min (p(x), 1 / 2)]} \frac{n}{k}\left(\frac{k}{n}-p(x)\right) b_{n k}(p(x))+\sum_{k=[n \min (p(x), 1 / 2)]}^{[n / 2]} b_{n k}(p(x)) \leq c,
\end{gathered}
$$

and since now $\varphi(x) \geq c$, Lemma 1 for $(\alpha, \beta)=(1 / 2,1),(3 / 2,2),(0,1)$ gives

$$
\begin{gathered}
B \leq c \sum_{k=1}^{[n / 2]}\left|x-x_{k t}\right|\left(1+\sqrt{\frac{n}{k}}\left|x-x_{k t}\right|\right) b_{n k}(p(x)) \\
\leq c \sum_{k=1}^{[n \min (p(x), 1 / 2)]}\left\{\sqrt{\frac{n}{k}}\left(p(x)-\frac{k}{n}\right)+\left(\frac{n}{k}\right)^{3 / 2}\left(p(x)-\frac{k}{n}\right)^{2}\right\} b_{n k}(p(x)) \\
+\sum_{k=[n \min (p(x), 1 / 2)]}^{[n / 2]}\left\{\frac{1}{\sqrt{p(x)}}\left(\frac{k}{n}-p(x)\right)\right\} b_{n k}(p(x)) \leq \frac{c}{\sqrt{n}} \leq c \frac{\varphi(x)}{\sqrt{n}} .
\end{gathered}
$$

Finally, using

$\left|\ell_{k i}(x)\right| \leq c\left|\frac{\left(x-x_{k t}\right)\left(x-x_{k, t-1}\right)}{x_{k i}-x_{k, i \pm 1}}\right| \leq c\left|x-x_{k t}\right|+c \sqrt{\frac{n}{k}}\left(x-x_{k t}\right)^{2}, \quad i \notin S$.

we get

$$
C \leq c \sum_{k=1}^{[n / 2]}\left(\left|x-x_{k t}\right|+\sqrt{\frac{n}{k}}\left(x-x_{k t}\right)^{2}\right) b_{n k}(p(x)) .
$$

But this leads to the same estimate as for $B$ above.

Next, we state an equivalence result.

Theorem 2. We have 
1 Bernstein-type polynomials on several intervals

$$
\left\|f-B_{n}(f)\right\|_{J_{s}}=O\left(n^{-\alpha / 2}\right) \Longleftrightarrow \omega_{\varphi}(f, t)=O\left(t^{\alpha}\right), \quad 0<\alpha<1 .
$$

For the proof we need two lemmas.

Lemma 2. We have

$$
\left\|\varphi B_{n}^{\prime}(f)\right\|_{J_{s}} \leq c \sqrt{n} V(f)
$$

for all $f \in C\left(J_{s}\right)$.

Proof of Lemma 2. We make the same assumptions on $x$ and on the summation for $k$ as in the proof of Theorem 1. Differentiating the identity

$$
\sum_{k=0}^{n} \sum_{i=1}^{m_{k}} \ell_{k i}(x) b_{n k}(p(x))=1
$$

we obtain

$$
\sum_{k=0}^{n} \sum_{i=1}^{m_{k}} f(x) \ell_{k i}(x) b_{n k}^{\prime}(p(x)) p^{\prime}(x)+\sum_{k=0}^{n} \sum_{i=1}^{m_{k}} f(x) \ell_{k i}^{\prime}(x) b_{n k}(p(x))=0 .
$$

Also, differentiating the operator $B_{n}(f, x)$ and subtracting the pervious relation we obtain

$$
\begin{gathered}
\varphi(x)\left|B_{n}^{\prime}(f, x)\right| \leq \varphi(x) V(f) \sum_{k=0}^{n} \sum_{i=1}^{m_{k}}\left\{\left|\ell_{k i}(x)\right| b_{n k}^{\prime}(p(x))\left|p^{\prime}(x)\right|+\left|\ell_{k i}^{\prime}(x)\right| b_{n k}(p(x))\right\} \\
\leq c V(f) \sum_{k=0}^{n} \sum_{i=1}^{m_{k}}\left\{\sqrt{p(x)}\left|\ell_{k i}(x)\right| b_{n k}^{\prime}(p(x))+\left|\ell_{k i}^{\prime}(x)\right| b_{n k}(p(x))\right\},
\end{gathered}
$$

since

$$
\varphi(x)\left|p^{\prime}(x)\right| \leq c \sqrt{p(x)} .
$$

Thus we have to prove:

$$
A:=\sqrt{p(x)} \sum_{k=0}^{[n / 2]}\left(\sum_{i=1}^{m_{k}}\left|\ell_{k i}(x)\right|\right) b_{n k}^{\prime}(p(x)) \leq c \sqrt{n}
$$

and

$$
B:=\sum_{k=0}^{[n / 2]}\left(\sum_{i=1}^{m_{k}}\left|\ell_{k i}^{\prime}(x)\right|\right) b_{n k}(p(x)) \leq c \sqrt{n} .
$$

For $k=0$ we have $\left|\ell_{k i}(x)\right| \leq c$, and by the Markov inequality $\left|\ell_{k i}^{\prime}(x)\right| \leq c$ for all $i=1, \ldots, m_{0}$, thus the contribution to $A$ and $B$ will be

$$
n \sqrt{p(x)}(1-p(x))^{n-1} \leq c \sqrt{n} \text { and } c(1-p(x))^{n} \leq c,
$$


respectively.

So let $1 \leq k \leq[n / 2]$. Since

$$
\left|b_{n k}^{\prime}(p(x))\right| \leq \frac{c n}{p(x)}\left|p(x)-\frac{k}{n}\right| b_{n k}(p(x)),
$$

we have

$$
A \leq c \sqrt{n}+\frac{c n}{\sqrt{p(x)}} \sum_{k=1}^{[n / 2]}\left(\sum_{i=1}^{m_{k}}\left|\ell_{k i}(x)\right|\right)\left|p(x)-\frac{k}{n}\right| b_{n k}(p(x)) .
$$

From the proof of Theorem 1 we can see that

$$
\begin{gathered}
\left|\ell_{k i}(x)\right| \leq c+c \sqrt{\frac{n}{k}}\left|x-x_{k t}\right| \\
\leq c+ \begin{cases}c \frac{n}{k}\left(p(x)-\frac{k}{n}\right), & \text { if } 1 \leq k \leq n \min (p(x), 1 / 2), \\
0, & \text { if } n \min (p(x), 1 / 2)<k \leq[n / 2],\end{cases}
\end{gathered}
$$

$i=1, \ldots, m_{k} ; k=1, \ldots,[n / 2]$, where again $x_{k t}$ is the nearest node to $x$ on the right. Thus we obtain, using Lemma 1 with $\alpha=1, \beta=2$,

$$
\begin{gathered}
A \leq c \sqrt{n}+\frac{c n}{\sqrt{p(x)}} \sum_{k=1}^{n \min (p(x), 1 / 2)} \frac{n}{k}\left(p(x)-\frac{k}{n}\right)^{2} b_{n k}(p(x)) \\
\leq c \sqrt{n}+\frac{c n}{\sqrt{p(x)}}\left(\sqrt{\frac{p(x)}{n}}+\frac{1}{n}\right) \leq c \sqrt{n},
\end{gathered}
$$

since the sum appears only if $p(x) \geq 1 / n$.

Finally, since $\left|\ell_{k i}(x)\right| \leq c \sqrt{n}$, consequently $\left|\ell_{k i}^{\prime}(x)\right| \leq c \sqrt{n}$, we obtain

$$
B \leq c \sqrt{n} \sum_{k=0}^{n} b_{n k}(p(x)) \leq c \sqrt{n} .
$$

Lemma 3. We have

$$
\left\|\varphi B_{n}^{\prime}(f)\right\|_{J_{s}} \leq c\left(\left\|\varphi f^{\prime}\right\|_{J_{s}}+V(f)\right)
$$

for all $f^{\prime} \in C\left(J_{s}\right)$.

Remark. The example of a piecewise constant function shows that the second term on the right hand side is necessary.

Proof of Lemma 3. Similarly as in the proof of Lemma 2, by (1.6) and (1.7) we obtain 


$$
\begin{aligned}
\varphi(x)\left|B_{n}^{\prime}(f, x)\right| \leq & c \varphi(x)\left|p^{\prime}(x)\right| \sum_{k=0}^{n}\left(\sum_{i=1}^{m_{k}}\left|f(x)-f\left(x_{k i}\right)\right| \cdot\left|\ell_{k i}(x)\right|\right)\left|b_{n k}^{\prime}(p(x))\right| \\
& +c \varphi(x) \sum_{k=0}^{n}\left(\sum_{i=1}^{m_{k}}\left|f(x)-f\left(x_{k i}\right)\right| \cdot\left|\ell_{k i}^{\prime}(x)\right|\right) b_{n k}(p(x)) .
\end{aligned}
$$

Since $\varphi(x) \geq \sqrt{x-a_{j}}$, we have

$$
\begin{gathered}
\varphi(x)\left|f(x)-f\left(x_{k i}\right)\right|=\varphi(x)\left|\int_{x_{k i}}^{x} f^{\prime}(t) d t\right| \\
\leq c \varphi(x)\left\|\varphi f^{\prime}\right\|_{J_{s}} \int_{x_{k i}}^{x} \frac{d t}{\varphi(t)} \leq c\left|x-x_{k i}\right| \cdot\left\|\varphi f^{\prime}\right\|_{J_{s}}, \quad i \in S_{k} .
\end{gathered}
$$

Hence (1.8) will take the form

$$
\begin{aligned}
& \varphi(x)\left|B_{n}^{\prime}(f, x)\right| \leq c\left\|\varphi f^{\prime}\right\|_{J_{s}} \sum_{k=0}^{n}\left\{\left(\sum_{i \in S_{k}}\left|\left(x-x_{k i}\right) \ell_{k i}(x)\right|\right) p^{\prime}(x)\left|b_{n k}^{\prime}(p(x))\right|\right. \\
&\left.+\left(\sum_{i \in S_{k}}\left|\left(x-x_{k i}\right) \ell_{k i}^{\prime}(x)\right|\right) b_{n k}(p(x))\right\} \\
&+c V(f) \varphi(x) \sum_{k=0}^{n}\left\{\left(\sum_{i \notin S_{k}}\left|\ell_{k i}(x)\right|\right) p^{\prime}(x)\left|b_{n k}^{\prime}(p(x))\right|+\left(\sum_{i \notin S_{k}}\left|\ell_{k i}^{\prime}(x)\right|\right) b_{n k}(p(x))\right\} .
\end{aligned}
$$

Let

$$
\begin{gathered}
A_{k}:=p^{\prime}(x) \sum_{i \in S_{k}}\left|\left(x-x_{k i}\right) \ell_{k i}(x)\right|, \quad B_{k}:=\sum_{i \in S_{k}}\left|\left(x-x_{k i}\right) \ell_{k i}^{\prime}(x)\right|, \\
C_{k}:=p^{\prime}(x) \varphi(x) \sum_{i \notin S_{k}}\left|\ell_{k i}(x)\right|, \quad D_{k}:=\varphi(x) \sum_{i \notin S_{k}}\left|\ell_{k i}^{\prime}(x)\right| .
\end{gathered}
$$

Let first $k=0$. Then $A_{0} \sim C_{0} \leq c\left(x-x_{0 t}\right)$, and both contribute

$$
\begin{gathered}
A_{0}\left|b_{n 0}^{\prime}(p(x))\right| \sim C_{0}\left|b_{n 0}^{\prime}(p(x))\right| \leq \\
\leq c n p^{\prime}(x)\left(x-x_{0 t}\right)(1-p(x))^{n-1} \leq \operatorname{cnp}(x)(1-p(x))^{n-1} \leq c .
\end{gathered}
$$

Similarly, $B_{0} \sim D_{0} \leq c$, and their contribution is also majorized by constant.

Now let $1 \leq k \leq n / 2$. Assume first that $a_{j}=\xi<x<\eta$ (i.e. $x$ is at the left "edge" of the interval $I_{j}$ ). Then

$$
A_{k} \sim B_{k} \leq c\left|p(x)-\frac{k}{n}\right|, \quad C_{k} \leq c \sqrt{\frac{n p(x)}{k}}\left|p(x)-\frac{k}{n}\right|, \quad D_{k} \sim \sqrt{\frac{n p(x)}{k}},
$$


Thus summing up for $1 \leq k \leq n$ using these estimates and the relation $\left|b_{n k}^{\prime}(x)\right| \leq c \frac{n}{x}\left|x-\frac{k}{n}\right| b_{n k}(x)$ we get

$$
\sum_{k=1}^{n} A_{k} b_{n k}^{\prime}(p(x)) \leq c \frac{n}{p(x)} \sum_{k=1}^{n}\left(p(x)-\frac{k}{n}\right)^{2} b_{n k}(p(x)) \leq c
$$

(cf. (1.5) with $\gamma=2$ ),

$$
\sum_{k=1}^{n} B_{k} b_{n k}(p(x)) \leq c \sum_{k=1}^{n}\left|p(x)-\frac{k}{n}\right| b_{n k}(p(x)) \leq c \sqrt{\frac{p(x)}{n}}
$$

(cf. (1.5) with $\gamma=1$ ),

$$
\begin{gathered}
\sum_{k=1}^{n} C_{k}\left|b_{n k}^{\prime}(p(x))\right| \leq c \frac{n}{p(x)} \sum_{k=1}^{n} \sqrt{\frac{n p(x)}{k}}\left(p(x)-\frac{k}{n}\right)^{2} b_{n k}(p(x)) \\
\leq \frac{c n}{\sqrt{p(x)}}\left(\sum_{k=1}^{n} \frac{k}{n}\left(p(x)-\frac{k}{n}\right)^{2} b_{n k}(p(x)) \sum_{k=1}^{n}\left(p(x)-\frac{k}{n}\right)^{2} b_{n k}(p(x))\right)^{1 / 2} \\
\leq \frac{c n}{\sqrt{p(x)}}\left(\frac{1}{n} \cdot \frac{p(x)}{n}\right)^{1 / 2} \leq c
\end{gathered}
$$

(cf. Lemma 1 with $\alpha=1, \beta=2$ ), and

$\sum_{k=1}^{n} D_{k} b_{n k}(p(x)) \leq c \sum_{k=1}^{n} \sqrt{\frac{n p(x)}{k}} b_{n k}(p(x)) \leq \sqrt{p(x)} \sum_{k=1}^{n}\left(\frac{n}{k} b_{n k}(p(x))\right)^{1 / 2} \leq c$

(cf. Lemma 1 with $\alpha=1, \beta=0$ ).

Finally, let $a_{j}<\xi<x<\eta$, i.e. $x$ is "inside" the interval $I_{j}$. Since now $p^{\prime}(x) \leq c \sqrt{p(x)}$, we have

$$
A_{k} \sim C_{k} \leq c \sqrt{p(x)}\left(\sqrt{\frac{n}{k}}\left(x-x_{k t}\right)^{2}+x-x_{k t}\right), \quad B_{k} \sim D_{k} \leq c \sqrt{\frac{n}{k}}\left(x-x_{k t}\right)+1 .
$$

Using previous estimates for $\left|x-x_{k t}\right|$ we get

$$
\begin{aligned}
& \sum_{k=1}^{n} A_{k} \mid b_{n k}^{\prime}\left(p(x)\left|\leq c \frac{n}{\sqrt{p(x)}} \sum_{k=1}^{n}\left(\sqrt{\frac{n}{k}}\left(x-x_{k t}\right)^{2}+x-x_{k t}\right)\right| p(x)-\frac{k}{n} \mid b_{n k}(p(x))\right. \\
& \leq \frac{c n}{\sqrt{p(x)}} \sum_{1 \leq k \leq n p(x)}\left[\left(\frac{n}{k}\right)^{3 / 2}\left|p(x)-\frac{k}{n}\right|^{3}+\sqrt{\frac{n}{k}}\left(p(x)-\frac{k}{n}\right)^{2}\right] b_{n k}(p(x))
\end{aligned}
$$




$$
\begin{gathered}
+\frac{c n}{p(x)} \sum_{n p(x) \leq k \leq n}\left(p(x)-\frac{k}{n}\right)^{2} b_{n k}(p(x)) \leq c n \sum_{k=1}^{n}\left(\frac{n}{k}\right)^{2}\left|p(x)-\frac{k}{n}\right|^{3} b_{n k}(p(x)) \\
+\frac{n}{\sqrt{p(x)}}\left(\sum_{k=1}^{n} \frac{n}{k}\left(p(x)-\frac{k}{n}\right)^{2} b_{n k}(p(x))\right)^{1 / 2}+c \\
\leq c n\left(\frac{1}{n}+\frac{1}{\sqrt{x} n^{3 / 2}}\right)+\frac{1}{\sqrt{n p(x)}}+c \leq c .
\end{gathered}
$$

And finally,

$$
\begin{gathered}
\sum_{k=1}^{n}\left[\sqrt{\frac{n}{k}}+1\right] b_{n k}(p(x)) \leq c \sum_{1 \leq k \leq n p(x)} \frac{n}{k}\left|p(x)-\frac{k}{n}\right| b_{n k}(p(x)) \\
+\sum_{n p(x) \leq k \leq n} b_{n k}(p(x))+c \leq c .
\end{gathered}
$$

Proof of Theorem 2. The implication " $\Longleftarrow$ follows from Theorem 1 . The proof of the other direction is modelled after the proof of (9.3.3) in [1] for the classic Bernstein-type operators. Define the $K$-functional by

$$
K_{\varphi}(f, t):=\inf \left(\|f-g\|_{J_{s}}+t\left\|\varphi f^{\prime}\right\|_{J_{s}}\right),
$$

where the infimum is taken over all functions $g$ which are absolutely continuous on each interval $I_{j}$. By applying Lemmas 2 and 3 for $f i g$ and $g$, respectively, we get

$$
\begin{aligned}
K_{\varphi}\left(f, \frac{1}{\sqrt{n}}\right) & \leq\left\|f-B_{k}(f)\right\|_{J_{s}}+\frac{1}{\sqrt{n}}\left\|\varphi B_{k}^{\prime}(f)\right\|_{J_{s}} \\
& \leq\left\|f-B_{k}(f)\right\|_{J_{s}}+\frac{c}{\sqrt{n}}\left(\sqrt{k}\|f-g\|_{J_{s}}+\left\|\varphi g^{\prime}\right\|_{J_{s}}+c\right) \\
& \leq\left\|f-B_{k}(f)\right\|_{J_{s}}+\frac{c}{\sqrt{k}}+c \sqrt{\frac{k}{n}} K_{\varphi}\left(f, \frac{1}{\sqrt{k}}\right) \\
& \leq \frac{c}{k^{\alpha / 2}}+c \sqrt{\frac{k}{n}} K_{\varphi}\left(f, \frac{1}{\sqrt{k}}\right), \quad 0<\alpha<1 .
\end{aligned}
$$

Applying the Berens-Lorentz lemma (cf. Lemma 9.3.4 from [1]) with $r=1 / 2$ we get

$$
K_{\varphi}\left(f, \frac{1}{\sqrt{n}}\right) \leq \frac{c}{n^{\alpha / 2}}, \quad \text { whence } \quad K_{\varphi}(f, t) \leq c t^{\alpha}, \quad 0<\alpha<1 .
$$

This easily implies $\omega_{\varphi}(f, t) \leq t^{\alpha}$ (cf. the proof in Section 2.4 in [1]). 
We do not know if the operator $B_{n}$ is saturated with $O\left(n^{-1 / 2}\right)$. The following example seems to support this conjecture.

Example. Let $m-s \geq 1$. Then there are $m-s$ extremal points of $p(x)$ inside $J_{s}$. Assume that there exists an extremal point such that $p(x)=1$, and let

$$
f_{0}(x)=\prod_{i=1}^{m_{n}}\left(x-x_{n i}\right) \in \Pi_{m_{n}}, \quad m_{n}=\left[\frac{m+s}{2}\right] .
$$

Then

$$
\left\|f_{0}(x)-B_{n}\left(f_{0}, x\right)\right\|_{J_{s}} \sim \frac{1}{\sqrt{n}} .
$$

Here the upper estimate follows from Theorem 1. To prove the lower estimate, note that

$$
L_{k}\left(f_{0}, x\right)= \begin{cases}f_{0}(x), & \text { if } \quad k=0, \ldots, n-1, \\ 0, & \text { if } \quad k=n\end{cases}
$$

Thus

$$
f_{0}(x)-B_{n}\left(f_{0}, x\right)=f_{0}(x) p(x)^{n} .
$$

Now let $x_{n r} \in \operatorname{int} J_{s}$ be such that $p\left(x_{n r}\right)=1$ and let $x=x_{n r}+\frac{1}{\sqrt{n}}$. Then

$$
\begin{gathered}
\left|f_{0}(x)-B_{n}\left(f_{0}, x\right)\right| \geq c\left(x-x_{n r}\right)\left(1-\left|p\left(x_{n r}\right)-p(x)\right|\right)^{n} \\
\geq c\left(x-x_{n r}\right)\left(1-c\left(x-x_{n r}\right)^{2}\right)^{n} \geq \frac{c}{\sqrt{n}} .
\end{gathered}
$$

\section{References}

1. Z. Ditzian and V. Totik, Moduli of Smoothness, Springer (1987).

2. A. Kroó and J. Szabados, Inverse polynomial mappings and Lagrange interpolation on several intervals, J. Math. Anal. Appl., 436 (2016), 1165-1179.

3. G. G. Lorentz, Bernstein Polynomials, Chelsea Publishing Company (New York, 1986).

4. A. L. Lukashov and J. Szabados, The order of Lebesgue constant of Lagrange interpolation on several intervals, Periodica Math. Hungar., DOI10.1007/s10998-015-0106-z

5. F. Peherstorfer, Orthogonal and Chebyshev polynomials on two intervals, Acta Math. Hungar., 55 (1990), 245-278.

6. F. Peherstorfer, K. Schiefermayr, Description of extremal polynomials on several intervals and their computation.I, Acta Math. Hungar., 83 (1999), 27-58. 